\title{
A INOVAÇÃO DAS NAÇÕES: UMA ANÁLISE SOB O VIÉS DA CULTURA NACIONAL
}

\author{
INNOVATION OF NATIONS: \\ AN ANALYSIS FROM THE BIAS OF NATIONAL CULTURE
}

JANDIR TAMBOSI JUNIOR

Universidade Regional de Blumenau (FURB)

Mestre em Administração (FURB)

Orcid: http://orcid.org/0000-0002-6556-8137 / E-mail: jrtambosi@gmail.com

Rua Antônio da Veiga, 140 - Itoupava Seca - CEP 89030-903 - Blumenau - SC

SILVANA SILVA VIEIRA TAMBOSI

Universidade Regional de Blumenau (FURB)

Doutoranda em Ciências Contábeis e Administração (FURB)

Orcid: http://orcid.org/0000-0002-4762-7957 / E-mail: profa.silvana.vieira@gmail.com

\author{
CHRISTIAN DANIEL FALASTER \\ Professor do PPG em Administração da Universidade Regional de Blumenau (PPGAD/FURB) \\ Doutor em Administração (FURB) \\ Orcid: http://orcid.org/0000-0001-9502-4475 / E-mail: christianfalaster@gmail.com
}

Submissão: 27/05/2020. Revisão: 23/03/2021. Aceite: 25/07/2021. Publicação: 28/07/2021.

DOI: http://dx.doi.org/10.22277/rgo.v14i3.5522

\begin{abstract}
RESUMO
A inovação é um dos principais fatores fomentadores do desenvolvimento econômico e social, tornando uma condição para que as nações prosperem em um contexto dinâmico e global. Portanto, saber quais fatores culturais implicam em um melhor desempenho de inovação fazse relevante. Assim, o objetivo que norteou esta pesquisa foi verificar a influência da cultura nacional sobre a inovação das nações. Para tal, utilizaram-se como procedimentos metodológicos a abordagem quantitativa com o uso da técnica descritiva e documental. $\mathrm{O}$ tratamento e análise dos dados foi realizado por meio da regressão linear múltipla. Para atender ao objetivo, o estudo embasou-se nos dados mais recentes dos sites de Hofstede INSIGHTS, que disponibiliza indicadores de cultura nacional os scores de inovação do The global Innovation Index, que apresentam dados de 66 países. Concluiu-se, a partir da análise dos resultados encontrados, que as hipóteses de que a distância do poder influencia negativamente a inovação dos países, devido à ausência da interação social em diferentes níveis hierárquicos, foi confirmada. Verificou-se também que, quanto maior o nível da cultura nacional do individualismo, maior é a capacidade de inovação da nação. Além disso, confirmou-se a hipótese de que a cultura nacional de incerteza diminui a capacidade de inovação da nação. Por outro lado, foram negadas as hipóteses de que quanto menor o índice de masculinidade maior seria a capacidade de inovação da nação, e que países com orientação de longo prazo e mais indulgentes teriam maior capacidade de inovação.
\end{abstract}

Palavras-chave: Cultura Nacional. Inovação. Inovação das Nações.

Este é um artigo publicado em acesso aberto (Open Access) sob a licença Creative Commons Attribution, que permite uso, distribuição e reprodução em qualquer meio, sem restrições desde que o trabalho original seja corretamente citado. 
A inovação das nações:

uma análise sob o viés da cultura nacional

\begin{abstract}
Innovation is one of the main drivers of economic and social development, making it a condition for nations to prosper in a dynamic and global context. Therefore, knowing which cultural factors imply a better innovation performance is relevant. Thus, the objective that guided this research was to verify the influence of the national culture on the nations' innovation. To this end, the quantitative approach was used as methodological procedures using the descriptive and documentary technique. Data treatment and analysis were performed using multiple linear regression. To answer the goal, the study was based on the most recent data from the Hofstede INSIGHTS websites, which provides national culture indicators with the innovation scores of The Global Innovation Index, which present data from 66 countries. From the analysis of the results found, the hypothesis that the distance from power negatively influences the innovation of the countries due to the absence of social interaction at different hierarchical levels was confirmed. It was also found that the higher the level of the national culture of individualism, the greater the nation's capacity for innovation. Also, the hypothesis was confirmed that the national culture of uncertainty diminishes the nation's ability for innovation. On the other hand, the hypotheses were denied that the lower the masculinity index, the greater the nation's capacity for innovation, and that countries with a long-term and more forgiving orientation would have a greater capacity for innovation.
\end{abstract}

Keywords: National Culture. Innovation. Nations Innovation.

\title{
1 INTRODUÇÃO
}

A inovação vem sendo tratada com muita atenção nos últimos tempos, pois, em um ambiente cada vez mais dinâmico e competitivo, este é um meio que as empresas encontram para responder à estas mudanças de forma rápida e assim, perdurarem sua existência neste ambiente.

O pioneiro acerca da inovação foi o economista Joseph Schumpeter em 1930, em que apontou a importância do tema e seu impacto nas organizações e no desenvolvimento econômico. Seus estudos evidenciaram vários tipos de inovações, como: inovação de produtos (novos produtos ou mudanças incrementais em produtos), inovação de processo (novos processos), etc. (SCHUMPETER, 1934). Estas tipologias vêm sendo cada vez mais estudadas pelas organizações, de modo a viabilizarem suas ideias de inovação.

A inovação é vista como um dos principais pontos que fomentam o desenvolvimento econômico e social, portanto, o assunto se torna relevante, pois, passa a ser uma condição para que as nações possam responder as mudanças com rapidez no cenário global (OECD, 2007). Um dos principais alvos das políticas econômicas das nações são o desenvolvimento econômico, que é influenciado por vários aspectos culturais/sociais (SHARIPOV, 2016). Diante deste cenário, estudos buscam identificar a diferença entre países e seus reflexos no desenvolvimento econômico das nações. Neste contexto, a inovação e a cultura nacional surgem como fatores do crescimento econômico.

A cultura nacional pode ser entendida como um meio necessário tanto para o crescimento quanto para o desenvolvimento econômico de uma nação, porém, não pode ser analisada de forma isolada, pois, também é necessário a existência de um contexto político e um mercado favorável que permita o seu desenvolvimento (HOFSTEDE; BOND, 1988). As mudanças culturais e o comportamento social são interligados, e acabam influenciando no 
desempenho de inovação e consequentemente nas decisões econômicas das nações (KOSTIS et al., 2018).

Algumas pesquisas vêm estudando esta relação entre cultura e inovação para o desenvolvimento econômico dos países. Da Silva e Moreira (2017), realizaram uma pesquisa relacionando a cultura nacional, medida pelas seis dimensões culturais de Hofstede, e o seu impacto na corrupção e inovação. Os resultados indicaram que a maioria dos aspectos da cultura nacional tem influência sobre a corrupção, embora seu impacto sobre a inovação seja menos mensurável. Os autores destacaram que a inovação é um motor para o crescimento e o desenvolvimento econômico, riqueza e prosperidade das nações.

Estudos da teoria institucional têm apresentado um conjunto de influências que os mesmos exercem sobre as características das organizações (MEYER; SCOTT; DEAL, 1983; TOLBERT; ZUCKER, 1983; SINGH, TUCKER; HOUSE, 1986; MEYER; SCOTT; STRANG, 1987; SCOTT, 1987) e sobre a mudança organizacional (HININGS; GREENWOOD, 1988). Estes estudos buscam explicar os fenômenos organizacionais, por meio das estruturas e processos organizacionais que se tornam legitimados e suas consequências nos resultados alcançados (SELZNICK, 1996; MEYER; ROWAN, 1977; ZUCKER, 1987; DANIELS; JOHNSON; CHERNATONY, 2002).

Diante deste contexto, saber quais são os fatores da cultura nacional implicam em um melhor desempenho de inovação se torna relevante. Assim, o objetivo que norteou este estudo foi: verificar a influência da cultura nacional sobre a inovação das nações. Para isso, são usados os Índices de Inovação Global (THE GLOBAL INNOVATION INDEX, 2017) e os dados mais recentes dos sites de Hofstede, que disponibiliza indicadores de cultura nacional (HOFSTEDE INSIGHT, 2017). Os dados longitudinais são analisados por meio de regressão linear múltipla, englobando os índices do período de 2017 de inovação global, e de 2015 referente a cultura nacional. Dessa forma, pretende-se contribuir com a teoria por meio da utilização de dados quantitativos de 66 países, para testar as hipóteses que relacionam essas variáveis, e a medida de sua correlação.

Nas próximas seções, é realizada a revisão da literatura, sobre inovação e cultura nacional. Na sequência, são descritos os procedimentos metodológicos usados no levantamento e análise dos dados. Posteriormente, é apresentada a análise e discussão dos resultados. Por fim, a última sessão compreende as principais conclusões e as referências utilizadas.

\section{REFERENCIAL TEÓRICO}

Nesta seção serão apresentados os principais tópicos relacionados a inovação e a cultura nacional.

\subsection{INOVAÇÃO}

A inovação é um tema relevante e que vem requerido muita atenção dos pesquisadores, gestores de empresas e muito discutido na agenda das nações. Isto se deve, pois, o ambiente social e econômico está cada vez mais dinâmico e competitivo. Em decorrência disto, as organizações encontram na inovação uma forma de responder de forma rápida a este ambiente caracterizado pela turbulência, e manterem competitivas no mercado.

O pioneiro a tratar da inovação foi o economista Joseph Schumpeter em 1930, no que Ihe concerne, destacou a importância da temática da inovação e seu efeito sobre as organizações, consequente, no desenvolvimento econômico. Lundvall (2017), segue a linha 
A inovação das nações:

uma análise sob o viés da cultura nacional

de definição de inovação de Schumpeter, em que é vista como "novas combinações", que por sua vez, pode ser separado da "invenção", que se torna uma inovação somente quando o empresário traz para o mercado. Lundvall adiciona ao conceito não apenas o evento da primeira introdução no mercado da nova combinação, mas também o processo de sua difusão e uso.

Os estudos de Schumpeter enunciaram também vários tipos de inovações, tais como: inovações de produtos, voltados ao desenvolvimento de novos produtos ou mudanças incrementais destes; a inovação de processos, destacando os novos processos que precisariam ou poderiam ser adotados nas empresas, entre outros. (SCHUMPETER, 1934). Segundo a Organisation for Economic Co-operation and Development - OECD (2007), a inovação é considerada como sendo um dos principais fatores fomentadores do desenvolvimento econômico e social, sendo uma condição para que as nações possam responder às mudanças com rapidez no cenário global.

Em decorrência disto, a temática da inovação vem sendo estudada em diversos campos do saber, tais como: engenharia, economia, negócios, ciência e sociologia (DAMANPOUR, 1992; GOPALAKRISHNANAN; DAMANPOUR; GOPALAKRISHNAN, 1997; O'SULLIVAN; DOOLEY, 2008; PRABHU; CHANDY, 2010). Com o crescente interesse acadêmico e sua importância na inserção da pauta econômica das nações, de acordo com Fagerber (2011), no fim dos anos de 1980 e no início dos anos de 1990, a literatura da inovação passou a fundamentar-se na política, direcionando-se a todos os agentes que influenciavam a inovação. Godin (2009), destaca que dentre os envolvidos no processo de inovação nacional, estão a indústria e seu ambiente, o governo e a universidade.

Neste sentido, tem-se a preocupação das nações no planejamento para o desenvolvimento nacional, e começou-se a discutir os sistemas nacionais de inovação. 0 sistema nacional de inovação foi introduzido na década de 1980 por Freeman (1987), Dosi et al. (1988), Lundvall (1992), Nelson (1993) e Edquist (1997). Vale ressaltar que não foi adotada nenhuma definição, com singularidade, para o sistema nacional de inovação. Conceitualmente, o sistema nacional de inovação é formado por um conjunto de instituições que produzem inovação, e com a intermediação do governo promovem a criação de políticas públicas que fomentem esta atividade (CHEN; GUAN, 2012). Ainda, nas políticas de inovação, o propósito é a competividade econômica tanto em países emergentes como em países em desenvolvimento (AGUIRRE-BASTOS; WEBER, 2017).

Nesta mesma linha de raciocínio, de acordo com Johnson, Edquist e Lundvall (2004). Existem duas direções que diferenciam a definição de sistemas nacionais de inovação. A primeira está relacionada ao contexto geográfico, e a segunda refere-se a um conjunto específico de tecnologia ou áreas de produto. Ademais, considerando uma abordagem mais ampla de sistemas nacionais de inovação, as instituições promotoras de inovação fazem parte de um sistema socioeconômico amplo, onde são compreendidas questões culturais, políticas e econômicas, além de auxiliarem na projeção de atividades inovadoras (FREEMAN, 2002).

Como é possível verificar, a inovação não se desenvolve de forma isolada, ou melhor, depende de uma integração de diferentes condições que possibilitam relacioná-la com o desenvolvimento econômico. Neste interim, têm-se as instituições e organizações que fazem parte da construção de competências no mercado de trabalho, na educação e na vida profissional (LUNDVALL, 2017). No estudo de Pradhan, Arvin e Bahmani (2018), foram analisados 49 países europeus nos anos de 1961 a 2014. Nestes, a inovação e o desenvolvimento financeiro foram considerados como sendo impulsionadores e essenciais, para a expansão econômica per capita à longo prazo destas nações pesquisadas. 
Jandir Tambosi Junior, Silvana Silva Vieira Tambosi e Christian Daniel Falaster

\subsection{CULTURA NACIONAL}

O conceito de cultura é proveniente da antropologia. Apesar de haver diversas definições do conceito de cultura, quando se trata do campo organizacional, busca-se uma concordância em relação às crenças, aos valores e os padrões que são externados socialmente nas atividades ou práticas organizacionais (HOFSTEDE et al. 1980). "A cultura é a programação coletiva da mente humana que distingue os membros de um grupo humano dos de outra". (HOFSTEDE, 1980, p. 24).

No contexto organizacional a cultura é constituída por um conjunto de símbolos, valores, práticas e artefatos que são compartilhadas pelas pessoas que fazem parte da organização. Pode-se citar a adoção de certos tipos de comportamentos, sendo considerados apropriados para aquele determinado grupo. Na maioria das vezes, estes comportamentos são recomendados pelos proprietários, fundadores da empresa. Com relação aos artefatos, inclui-se: as normas, os regulamentos, o layout físico. Considera-se também a forma pela qual as pessoas, nas instituições, se comportam entre elas, além dos artefatos físicos, tais como: documentos, em geral, relatórios, produtos, etc. (SCHEIN, 1990).

Outro aspecto interessante da cultura nacional, no contexto das organizações, é a sua influência a respeito de diversos aspectos, no que tange a configuração organizacional. Como exemplo, pode-se citar a estrutura, o modo como os gestores e demais colaboradores tomam decisões e resolvem problemas, e as suas expectativas (HOFSTEDE et al,1980). Pettigrew (1982) e Whittington (2006), corroboram ao afirmarem que as pessoas que compõem uma organização compartilham e reproduzem regras culturais, linguagens e procedimentos que conduzem e norteiam o comportamento de todo o grupo. Assim sendo, a cultura influência a forma como as pessoas se comunicam umas com as outras, compartilham conhecimentos e aprendizados em todos os setores da organização.

Neste sentido, entende-se que a cultura de uma organização institui um padrão de pressupostos trazidos como básicos, criados por grupos que, inicialmente, foram preconizados pelos fundadores e outros membros de influência da empresa. Estes formam um processo de socialização, no qual, compartilham e ensinam suas suposições e conjeturas sobre o mundo aos novos membros. O objetivo é apresentar princípios que admite a empresa a aprender a tratar os aspectos relacionados a adaptação ao ambiente externo, e desenvolver a concordância do grupo (BERGER; LUCKMAN,1967; SCHEIN,1984).

Para a mensuração da cultura, neste artigo foram adotados as dimensões e indicadores da cultura nacional de Hofstede (1980), tais como: distância de poder, individualismo, masculinidade, incerteza, orientação de longo prazo e indulgência. A primeira dimensão trata da distância de poder, esta dimensão está relacionada ao grau de desigualdade de uma nação, entre os níveis hierárquicos organizacionais.

Conforme Hofstede (2011), os membros de uma sociedade que possuem menos poder, permitem que o poder seja distribuído de forma desigual. As sociedades, em um modo geral, tendem a ser desiguais, entretanto, algumas podem ser mais desiguais do que outras sociedades. Um aspecto interessante é que, para as nações em que a distância de poder é baixa, os pais se relacionam com as crianças de forma igualitária, no entanto, em nações que apresentam maior distância do poder, os pais ensinam a suas crianças a obediência.

Algumas características desta dimensão são: as deliberações sobre decisões são tomadas sem a consulta de pessoas em posições inferiores na hierarquia; pessoas que possuem posições superiores devem evitar a interação social com pessoas em posições inferiores; e as pessoas em posições inferiores na hierarquia, devem obedecer às decisões tomadas por pessoas em níveis superiores na hierarquia (HOFSTEDE, 1980). Altos níveis de 
A inovação das nações:

uma análise sob o viés da cultura nacional

centralização e formalização foram encontrados associados a taxas mais baixas de adoção de inovação (ZMUD, 1982). Diante destas características estabelece-se:

$\mathrm{H}_{1}$ : Quanto maior a distância de poder menor é a capacidade de inovação da nação.

A segunda dimensão é o individualismo, este trata-se de um indicador caracterizado pelo individualismo e coletivismo. No que tange o coletivismo, as nações em que a cultura nacional dominante é o coletivismo, é priorizada a integração entre os grupos, e o relacionamento está acima da tarefa a ser realizada. Em países em que a cultura nacional é individualista, prevalece o direito à privacidade (HOFSTEDE, 2011). Algumas características desta dimensão são: o trabalho em grupo é estimulado pela liderança; decisões são tomadas em grupo; os resultados são avaliados pela participação do grupo (HOFSTEDE, 1980).

Relacionando esta dimensão (individualismo) com o desenvolvimento de uma nação, Couto e Vieira (2004), explanam que um país em que a cultura nacional predominante é o individualismo, o nível de atividades de pesquisa e desenvolvimento tendem a ser maiores do que em países em que a cultura nacional é o coletivismo. Fazendo com que a emissão de patentes seja mais frequentemente concedida à países individualistas do que em coletivistas (Hofstede, 2001). Sendo assim estabelece-se:

$\mathrm{H}_{2}$ : Quanto maior o nível de individualismo, maior é a capacidade de inovação da nação.

Em seguida, tem-se a dimensão masculinidade, que realça a separação de funções pelos sexos masculinos e femininos. Nações com baixos índices para este indicador demonstra que: homens e mulheres possuem o poder e o sucesso de forma mais igualitária. Algumas características desta dimensão são: um alto nível para este indicador representa que determinadas atividades são realizadas pelo sexo feminino, e outras pelo sexo masculino, e a alternância destes papeis podem ser malvistos pela sociedade. O poder é distribuído independente do sexo (HOFSTEDE, 1980). Diante deste contexto estabelece-se:

$\mathrm{H}_{3}$ : Quanto menor o índice de masculinidade maior é a capacidade de inovação da nação.

A outra dimensão é a incerteza, este indicador apresenta as situações duvidosas ou incertas dos países. Um alto índice para este indicador demonstra leis duras e bastante formalismo nos negócios, além de uma sociedade bastante regrada refletindo em restrições a inovações. As características desta dimensão são: o formalismo nos negócios, estruturas organizacionais fortes, e furtar-se de riscos (HOFSTEDE, 1980). Diante deste cenário estabelece-se:

$\mathrm{H}_{4}$ : Quanto maior a incerteza menor a capacidade de inovação da nação.

A penúltima dimensão é a orientação a longo prazo, esta equivale à busca por resultados no longo prazo, e são consideradas menos criativa, onde respeitam as tradições e a cultura de seus ancestrais, portanto, são vistas como mais tradicionais. Algumas características desta dimensão são: a família, como sendo a base da sociedade, as pessoas de mais idade possuem mais respeito do que os jovens, e as mudanças de regras são restritas. Empresas em culturas nacionais com uma orientação de longo prazo, são mais receptivas a investimentos profundos em mudanças de longo prazo, e se concentram em resultados futuros (HOFSTEDE, 1980). Portanto, diante destas características estabelece-se:

$\mathrm{H}_{5}$ : Quanto maior o índice de orientação de longo prazo maior é a capacidade de inovação da nação. 
Por último, tem-se a dimensão indulgência, esta aponta como a sociedade trata seus impulsos e desejos. Um alto grau para este indicador demonstra uma sociedade que busca a felicidade, desejos e o prazer de seus impulsos. Algumas características desta dimensão são: a busca da felicidade e alegria e a pouca ansiedade com prazos (HOFSTEDE, 1980). Quando a cultura nacional da indulgência não é proeminente em uma sociedade, esta é caracterizada pela restrição, assim, este país adota normas sociais rígidas. Em uma nação em que os índices de indulgência são sobressalentes, o lazer é visto como um aspecto importante na vida das pessoas. Por outro lado, em que os índices de indulgência não são altos, têm-se mais restrições, e diminui-se a importância do lazer para aquela sociedade (HOFSTEDE, 2011). Diante deste contexto estabelece-se:

$\mathrm{H}_{6}$ : Quanto maior a indulgência maior a capacidade de inovação da nação.

Ao relacionar a cultura nacional de um país com a inovação, Hofstede e Bond (1988), afirmam que a cultura nacional é abalizada como sendo uma condição essencial no desenvolvimento econômico de um país. Embora não seja um elemento que deve ser examinado de forma isolada, visto que para o desenvolvimento econômico de um país faz-se necessário um mercado justo e favorável, em um panorama político que promova o seu desenvolvimento (HOFSTEDE; BOND, 1988). Ainda, de acordo com Kostis et al. (2018), as mudanças culturais estão relacionadas ao comportamento social, no que lhe concerne, influencia o desempenho inovador de uma nação, derivando em decisões econômicas.

É importante elucidar que a relação da cultura nacional e o comportamento dos indivíduos, pode ser analisada em situações em que as instituições e os países buscam investigar as possíveis falhas obtidas nas tomadas de decisões (ANGELIS, 2016). Bandarin et al. (2011), complementam ao apontar que, a cultura nacional configura um aspecto influenciador na geração de emprego e renda da sociedade.

\section{PROCEDIMENTOS METODOLÓGICOS}

A pesquisa classifica-se como descritiva em relação ao objetivo, por apresentar planos estruturados com o objetivo de descrever características de uma amostra por meio de uma pergunta de pesquisa (HAIR JR. et al. 2005). Este estudo teve como objetivo verificar a influência da cultura nacional sobre a inovação das nações. Para isso, foi preciso analisar a cultura nacional sob o score de inovação de 66 nações. Quanto a abordagem, classifica-se como quantitativa, uma vez que é uma das formas de verificar a relação existente entre as variáveis com as teorias de inovação e cultura nacional. Desta forma, as variáveis e os seus respectivos dados podem ser testados estatisticamente (CRESWELL, 2010). Quanto a técnica de análise estatística, para esta pesquisa optou-se pelo método de regressão linear múltipla, a fim de analisar as influências da cultura nacional sobre a inovação.

O estudo classifica-se também como documental, visto que foram utilizados dados do site do Índice Global de Inovação (2017) e do Hofstede Insight (2017), que apresentam indicadores de cultura nacional. Para que fosse possível relacionar as variáveis de cultura nacional e inovação, os dados dispostos nos sites foram compilados em uma planilha única de dados que permitiu a interação entre as informações disponíveis em ambos os bancos de dados.

Foram analisadas 66 nações por possuírem todas as informações necessárias para a realização deste estudo. Estes países foram: a Albânia, Argentina, Australia, Austria, Bangladesh, Belgium, Brazil, Bulgaria, Burkina Faso, Canada, Chile, China, Colombia, Croatia, Czech Republic, Denmark, Dominican Republic, Egypt, El Salvador, Estonia, Finland, France, 
A inovação das nações:

uma análise sob o viés da cultura nacional

Germany, Greece, China, Hungary, Iceland, India, Indonesia, Iran, Islamic Republic of Ireland, Italy, Japan, Jordan, Latvia, Lebanon, Lithuania, Malaysia, Mexico, Morocco, Mozambique, Netherlands, New Zealand, Nigeria, Norway, Peru, Philippines, Poland, Portugal, Romania, Russian Federation, Saudi Arabia, Serbia, Slovakia, Slovenia, South Africa, Spain, Sweden, Switzerland, Tanzania, United Republic of Thailand, Turkey, Ukraine, United Kingdom, United States of America Uruguay. Também foram utilizadas variáveis de controle como o PIB per capita, PIB e Market capitalization das nações, retiradas do site do Banco Mundial (2018).

No Quadro 1, apresenta-se o indicador que descreve as variáveis dependentes da inovação. Trata-se de dados do relatório do Índice de Inovação Global referente ao ano de 2017, isso dá-se ao fato de ser o relatório mais recente, quando da realização deste estudo. 0 Índice de Inovação Global é considerado referência em inovação nos últimos 10 anos, pois, possui um rico banco de dados de métricas detalhadas das nações. Este índice, no ano de 2017, abrangeu 127 países, que representam 92,5\% da população mundial e 97,6\% do PIB global (GII, 2017).

Quadro 1 - Indicador de inovação das nações

\begin{tabular}{|c|l|}
\hline Indicador & \multicolumn{1}{c|}{ Descrição do indicador } \\
\hline Score Inovação & $\begin{array}{l}\text { Score de Inovação é uma métrica de avaliação para examinar as inovações das } \\
\text { nações. }\end{array}$ \\
\hline
\end{tabular}

Fonte: Índice de Inovação Global (2018).

No Quadro 2 são apresentados os indicadores da cultura nacional que figuram as variáveis independentes deste estudo. Trata-se de dados do ano de 2015 , que são os mais atualizados disponibilizados no site Hofstede Insight (2017). Utilizou-se esta fonte de dados pelo acesso livre, e por disponibilizar as dimensões necessárias ao estudo. Ainda, esta homepage tem como intuito dar continuidade ao trabalho do Professor Geert Hofstede, que realiza diversas atividades acadêmicas e culturais, e é considerado um dos principais representantes de estudos e pesquisas interculturais em diversos países (HOFSTEDE INSIGHTS, 2017).

Quadro 2 - Dimensões e indicadores da cultura nacional

\begin{tabular}{|c|c|}
\hline Indicadores & Descrição do indicador \\
\hline $\begin{array}{l}\text { Distância de } \\
\text { Poder }\end{array}$ & $\begin{array}{l}\text { Este indicador apresenta o grau de desigualdade entre os níveis hierárquicos } \\
\text { organizacionais, números elevados para este indicador demonstra que a sociedade possui } \\
\text { maior nível hierárquico e desigual, logo, um número baixo para este indicador demonstra } \\
\text { que a sociedade é mais igualitária. Algumas características desta dimensão são: } \\
\text { - As deliberações sobre decisões são tomadas sem a consulta de pessoas em } \\
\text { - posições inferiores na hierarquia; } \\
\text { Pessoas nas posições hierárquicas superiores devem evitar a interação social com } \\
\text { - Pessoas em posições hierárquicas inferiores; } \\
\text { Pessoas em posições inferiores na hierarquia devem obedecer às decisões } \\
\text { tomadas por pessoas em níveis superiores na hierarquia. }\end{array}$ \\
\hline Individualismo & $\begin{array}{l}\text { Trata-se de um indicador de individualismo e coletivismo das nações. } \\
\text { Nações com baixo grau de individualismo tendem a privilegiar o coletivo. Algumas } \\
\text { características desta dimensão são: } \\
\text { - O trabalho em grupo é estimulado pela liderança; } \\
\text { - Decisões são tomadas em grupo; } \\
\text { - Resultados são avaliados pela participação do grupo. }\end{array}$ \\
\hline Masculinidade & $\begin{array}{l}\text { Corresponde a um indicador que apresenta a separação de funções por sexo: masculinos } \\
\text { e femininos. Nações com baixos índices para este indicador demonstra que, homens e } \\
\text { mulheres, possuem o poder e sucesso de forma mais igualitária. Algumas características } \\
\text { desta dimensão são: }\end{array}$ \\
\hline
\end{tabular}


Jandir Tambosi Junior, Silvana Silva Vieira Tambosi e Christian Daniel Falaster

\begin{tabular}{|c|c|}
\hline & $\begin{array}{l}\text { - Um alto nível para este indicador representa que determinadas atividades são } \\
\text { realizadas pelo sexo feminino e outra pelo sexo masculino, e a alternância destes } \\
\text { papeis pode ser malvisto pela sociedade. } \\
\text { - O poder é distribuído independente do sexo. }\end{array}$ \\
\hline Incerteza & $\begin{array}{l}\text { Representa o indicador do grau de ansiedade da nação em torno de situações duvidosas } \\
\text { ou incertas. Um alto índice para este indicador demonstra leis duras e bastante formalismo } \\
\text { nos negócios, além de uma sociedade bastante regrada. As características desta dimensão } \\
\text { são: } \\
\text { - Formalismo nos negócios; } \\
\text { - Estruturas organizacionais fortes; } \\
\text { - Evitam riscos. }\end{array}$ \\
\hline $\begin{array}{l}\text { Orientação de } \\
\text { longo prazo }\end{array}$ & $\begin{array}{l}\text { Trata-se do indicador de resultados de longo prazo. São consideradas nações menos } \\
\text { criativas, onde respeitam as tradições e a cultura de seus ancestrais, portanto, são vistas } \\
\text { como mais tradicionais. Algumas características desta dimensão são: } \\
\text { - Família como base da sociedade; } \\
\text { - Pessoas de mais idade possuem mais respeito que os jovens; } \\
\text { - Mudança de regras são restritas. }\end{array}$ \\
\hline Indulgência & $\begin{array}{l}\text { Refere-se a um indicador de como a sociedade trata seus impulsos e desejos. Um alto grau } \\
\text { para este indicador demonstra uma sociedade que busca a felicidade, desejos e o prazer } \\
\text { de seus impulsos. Algumas características desta dimensão são: } \\
\text { - Busca da felicidade e alegria; } \\
\text { - Pouca ansiedade com prazos. }\end{array}$ \\
\hline
\end{tabular}

Fonte: Hofstede (1980).

As dimensões de Hofstede tem sido fonte de estudos anteriores, envolvendo diversas áreas, tais como o empreendedorismo (ZHAO et al., 2012; OSTAPENKO, 2015), os sistemas financeiros (KWOK; TADESSE, 2006), a pesquisa e desenvolvimento (COUTO; VIEIRA, 2004), a atividade empresarial e o comércio internacional (BUSZKO, 2017; KRISTJÁNSDÓTTIR et al., 2017) e os benefícios privados de controle (SALZMANN; SOUPAK, 2017).

Depois de definida as dimensões da pesquisa e a fonte de informações, prosseguiu-se com a coletados dos dados, e utilizou-se o software Microsoft Excel ${ }^{\circledR}$ para tabular esses dados. Posteriormente, utilizou-se o software estatístico SPSS ${ }^{\circledR}$ versão 22 , para verificar como as variáveis se relacionavam. Para isso, foi realizada a análise estatística de regressão linear múltipla. Para Fávero et al. (2009) a regressão linear múltipla tem por objetivo identificar a relação explicativa entre duas ou mais variáveis, que se apresentam de forma linear. A regressão linear múltipla é representada modelo matemático conforme a equação:

$$
Y_{j} \widehat{Y}=\beta_{o}+\beta_{1} X_{1 j}+B_{2} X_{2 j}+\cdots+\beta_{p} X_{p j}+\epsilon_{j}(j=1, \ldots, n) \text {. }
$$

Para analisar a influência das variáveis de cultura nacional das nações sobre o índice de inovação utilizou-se o modelo 1: em que:

$$
\widehat{\mathrm{Y}}=\beta_{o}+\beta_{1}(D P)+B_{2}(I)+B_{3}(M)+B_{4}(I N C)+B_{5}(O L P)+B_{6}(I N D)+\varepsilon
$$

$$
\begin{aligned}
& \text { DP = Distância de poder } \\
& \text { I = Individualismo } \\
& M=\text { Masculinidade } \\
& \text { INC = Incerteza } \\
& \text { OLP = Orientação a longo prazo } \\
& \text { IND = Indulgência }
\end{aligned}
$$

Sendo assim, a utilização da técnica de análise estatística de regressão linear múltipla, permitiu verificar quais variáveis independentes poderiam influenciar a variável dependente. O estudo teve por objetivo avaliar a relação para cada variável, de forma individual com as variáveis de controle. A seguir, apresenta-se a análise e discussão dos resultados deste estudo. 
A inovação das nações:

uma análise sob o viés da cultura nacional

\section{ANÁLISE DOS RESULTADOS}

Esta seção tem o propósito de discutir os resultados encontrados na pesquisa. Para tal, realizou-se oito análises de regressão linear múltipla, objetivando verificar a influência da cultura nacional sobre a inovação das nações. Na Tabela 1, são apresentadas as correlações entre todas as variáveis propostas no estudo, e suas respectivas significâncias.

Tabela 1 - Tabela de correlações

\begin{tabular}{l|c|c|c|c|c|c|c|c|c|c}
\hline & 1 & 2 & 3 & 4 & 5 & 6 & 7 & 8 & 9 & 10 \\
\hline Score Innovation & 1,000 & & & & & & & & & \\
Distância de Poder &,$- 646^{* *}$ & 1,000 & & & & & & & & \\
Individualismo &, $709 * *$ &,$- 694 * *$ & 1,000 & & & & & & & \\
Masculinidade &,- 022 &, 093 &, 100 & 1,000 & & & & & & \\
Incerteza &,- 203 &, 200 &,- 218 &,- 129 & 1,000 & & & & & \\
Orientação Longo Prazo &, $499 *$ &,- 037 &, $253^{*}$ &, 026 &, 116 & 1,000 & & & & \\
Indulgência &, 229 &,$- 409^{* *}$ &, $247^{*}$ &, 038 &,- 204 &,$- 416^{* *}$ & 1,000 & & & \\
PIB PER CAPITA &, $906 * *$ &,$- 718^{* *}$ &, $718^{* *}$ &,- 013 &,- 113 &, $327^{* *}$ &, $347^{* *}$ & 1,000 & & \\
PIB &, $421^{* *}$ &,- 190 &, $371^{* *}$ &, $326 * *$ &,- 122 &, 223 &, $284^{*}$ &, $356^{* *}$ & 1,000 & \\
Marketcapitalization &, $363^{* *}$ &,- 107 &, 164 &,- 024 &,$- 261 *$ &, $422^{* *}$ &,- 093 &, 200 &, 188 & 1,000 \\
\hline
\end{tabular}

**. A correlação é significativa no nível 0,01 (2 extremidades).

* . A correlação é significativa no nível 0,05 (2 extremidades).

Fonte: dados da pesquisa.

A Tabela 2 apresenta a matriz de correlação para os dados, para a Regressão Linear Múltipla. Verificou-se que não há correlações altas que apontam multicolinearidade, pois, o fator de influência da variância (VIF), que mede o efeito das variáveis independentes sobre o coeficiente de regressão, ficaram abaixo de 5 . Uma estatística VIF igual ou maior que 5, indica a existência de multicolinearidade entre as variáveis explicativas do modelo proposto (FÁVERO et al., 2009). A existência de multicolinearidade significa que as variáveis independentes possuem correlação entre si, ou seja, a análise de regressão pode ser insignificante e confusa (MAROCO, 2003).

Tabela 2 - Resultados da Regressão Linear Múltipla para variáveis de Inovação

\begin{tabular}{|c|c|c|c|c|c|c|c|c|c|}
\hline & Dimensões & Mod.1 & Mod.2 & Mod. 3 & Mod. 4 & Mod. 5 & Mod. 6 & Mod. 7 & Mod. 8 \\
\hline \multirow{12}{*}{ 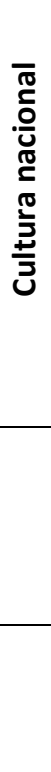 } & $\begin{array}{l}\text { Distância de } \\
\text { Poder }\end{array}$ & & $-0,160 * *$ & & & & & & $-0,098 * *$ \\
\hline & Individualismo & & & $0,164^{* *}$ & & & & & 0,065 \\
\hline & Masculinidade & & & & $-0,076$ & & & & $-0,081$ \\
\hline & Incerteza & & & & & $-0,164 * *$ & & & $-0,164 * *$ \\
\hline & $\begin{array}{l}\text { Orientação } \\
\text { Longo Prazo }\end{array}$ & & & & & & $0,103^{*}$ & & $0,206 * *$ \\
\hline & Indulgência & & & & & & & $-0,015$ & 0,036 \\
\hline & PIB Per Capita & $0,825^{* * *}$ & $0,712 * * *$ & $0,716^{* * *}$ & $0,811^{* * *}$ & $0,825^{* * *}$ & $0,797 * * *$ & $0,828 * * *$ & $0,633^{* * *}$ \\
\hline & PIB & 0,058 & 0,077 & 0,046 & 0,087 & 0,51 & 0,056 & 0,060 & 0,080 \\
\hline & $\begin{array}{l}\text { Market } \\
\text { Capitalization }\end{array}$ & $0,213 * * *$ & $0,210 * * *$ & $0,196 * * *$ & $0,207 * * *$ & $0,170 * *$ & $0,178 * *$ & $0,211 * * *$ & 0,089 \\
\hline & Adjusted $\mathrm{R}^{2}$ & 0,831 & 0,843 & 0,842 & 0,834 & 0,855 & 0,837 & 0,828 & 0,882 \\
\hline & $F$ & $107,542 * * *$ & $88,498 * * *$ & $87,478 * * *$ & $82,413 * * *$ & $96,741 * * *$ & $84,331 * * *$ & $79,471 * * *$ & $55,135^{* * *}$ \\
\hline & $\mathrm{N}$ & 65 & 65 & 65 & 65 & 65 & 65 & 65 & 65 \\
\hline
\end{tabular}

Fonte: dados da Pesquisa 
Ainda, na Tabela 2 foi calculada a regressão linear múltipla. O Modelo 1 considera as variáveis de controle, o Modelo 2 considera as variáveis independentes "distância de poder" e variáveis de controle, o Modelo 3 considera as variáveis independentes "individualismo" e variáveis de controle, o Modelo 4 considera as variáveis independentes "masculinidade" e variáveis de controle, o Modelo 5 considera as variáveis independentes "incerteza" e variáveis de controle, o Modelo 6 considera as variáveis independentes "Orientação Longo Prazo" e variáveis de controle, o Modelo 7 considera as variáveis independentes "Indulgência" e variáveis de controle, e o Modelo 8 apresenta o modelo completo, considerando todas as interações.

Visando validar a possibilidade de utilização da análise de regressão linear múltipla para este estudo, aplicou-se o teste ANOVA. Os parâmetros para este teste de regressão em conjunto devem ser iguais a zero, caracterizando-se desta forma uma relação estatisticamente significativa. Em todos os modelos o teste ANOVA foi de 0,000, possibilitando assim seguir com a análise. Na Tabela 3 são apresentadas as declarações das hipóteses.

Tabela 3 - Declaração das Hipóteses

\begin{tabular}{c|l|c}
\hline Hipótese & \multicolumn{1}{|c|}{ Declaração da Hipótese } & \multicolumn{1}{c}{ Status } \\
\hline $\mathrm{H}_{1}$ & $\begin{array}{l}\text { Quanto maior a distância de poder menor é a capacidade de inovação } \\
\text { da nação. }\end{array}$ & Confirmada \\
\hline $\mathrm{H}_{2}$ & $\begin{array}{l}\text { Quanto maior o nível de individualismo, maior é a capacidade de inovação } \\
\text { da nação. }\end{array}$ & Confirmada \\
\hline $\mathrm{H}_{3}$ & $\begin{array}{l}\text { Quanto menor o índice de masculinidade maior é a capacidade de inovação } \\
\text { da nação. }\end{array}$ & Não Confirmada \\
\hline $\mathrm{H}_{4}$ & Quanto maior a incerteza menor a capacidade de inovação da nação. & Confirmada \\
\hline $\mathrm{H}_{5}$ & $\begin{array}{l}\text { Quanto maior o índice de orientação de longo prazo maior é a capacidade } \\
\text { de inovação da nação. }\end{array}$ & Não Confirmada \\
\hline $\mathrm{H}_{6}$ & Quanto maior a indulgência maior a capacidade de inovação da nação. & Não Confirmada \\
\hline
\end{tabular}

Fonte: dados da Pesquisa

A partir da Tabela 3, pode-se verificar que a Hipótese 1 propunha que quanto maior a distância de poder menor é a capacidade de inovação da nação. Testou-se a primeira hipótese a partir do índice de "distância de poder" em relação ao score de "inovação das nações". 0 resultado do Modelo 2 mostra o $\beta$ negativo e significante $(\beta=-0.160, p<0.05)$. Confirmando assim a hipótese 1, ou seja, a "distância de poder" influencia negativamente a "inovação das nações". Infere-se que a distância do poder influencia negativamente a inovação dos países pela ausência da interação social em diferentes níveis hierárquicos, conforme Hofstede (1980). Além disso, Zmud (1982), complementarmente afirma que os altos níveis de centralização e formalização estão associados a taxas mais baixas de inovação. Os autores Zhao et al. (2012) e Ostapenko (2015) verificaram em seus estudos que, a distância do poder tem impacto no desenvolvimento de novos empreendimentos nas nações, devido à influência sociocultural do ambiente. Além disso, a distância do poder clarifica divergências na quantidade de atividades inovativas entre os países. Salzmann e Soupak (2017), também verificaram em sua pesquisa que a distância do poder está positivamente relacionada aos benefícios privados de controle.

A Hipótese 2 sugere que quanto maior o nível de individualismo, maior é a capacidade de inovação da nação. Testou-se a segunda hipótese, a partir do índice de "individualismo" em relação ao score de "inovação das nações". O resultado do Modelo 3 mostra o $\beta$ positivo e significante $(\beta=0.164, p<0.05)$. Confirmando assim a hipótese 2 , isto é, o individualismo influencia positivamente a inovação das nações. Este dado corrobora com o que afirmam 
A inovação das nações:

uma análise sob o viés da cultura nacional

Couto e Vieira (2004), segundo os autores em uma nação em que a cultura nacional é caracterizada pelo individualismo, as atividades de pesquisa e desenvolvimento tendem a ser maiores do que em países coletivistas. Além disso, segundo Hofstede (2001), a emissão de patentes é mais frequentemente concedida em países culturalmente individualistas do que os coletivistas.

A Hipótese 3 preconizava que quanto menor o índice de masculinidade maior seria a capacidade de inovação da nação. Testou-se a terceira hipótese, a partir do índice de masculinidade em relação ao score de inovação das nações. O resultado do Modelo 4 mostra o $\beta$ positivo e não significante $(\beta=-0.076, p>0.1)$. Não suportando assim a hipótese 3 . Em países em que, homens e mulheres possuem o poder de forma mais igualitária, o índice de inovação apresentou ser menor. Com base em Hofstede (1980) infere-se que talvez a separação das atividades por sexo (algumas são realizadas pelo sexo feminino, e outras pelo sexo masculino), e a alternância destes papeis, na maioria dos países investigados, podem ter contribuído para este resultado. No entanto, é pertinente que as nações estejam atentas a esta dimensão, pois, segundo Buszko (2017) e Kristjánsdóttir et al. (2017), a influência da cultura nacional sobre a masculinidade afeta as atividades comerciais, tanto nacionais quanto internacionais dos países.

A Hipótese 4 propunha que quanto maior a incerteza menor a capacidade de inovação da nação. Testou-se a quarta hipótese a partir do índice de incerteza em relação ao score de inovação das nações. $O$ resultado do Modelo 5 mostra o $\beta$ negativo e significante $(\beta=-0.164$, $p<0.05)$, confirmando esta hipótese. Supõe-se que os países investigados apresentam leis rígidas, além da presença de um forte formalismo nos negócios, característico de uma sociedade bastante regrada, o que reflete em restrições a inovações (HOFSTEDE, 1980).

A Hipótese 5 sugere que quanto maior o índice de orientação de longo prazo maior será a capacidade de inovação das nações. Testou-se a quinta hipótese a partir do índice de orientação de longo prazo em relação ao score de inovação das nações. O resultado do Modelo 6 mostra o $\beta$ positivo e não significante $(\beta=0.103, p<0.1)$. Não suportando assim hipótese 5. De acordo com Hofstede (1980), culturas nacionais em que a orientação é a longo prazo são consideradas menos criativa e mais tradicionais, e as mudança de regras são restritas. Além disso, as organizações estão mais propensas a investimentos densos e se concentram em resultados futuros (HOFSTEDE, 1980).

Por fim, na Hipótese 6 formulou-se que quanto maior a indulgência maior a capacidade de inovação da nação. Testou-se a sexta hipótese, a partir do índice de indulgência em relação ao score de inovação das nações. $O$ resultado do Modelo 7 mostra o $\beta$ negativo e não significante $(\beta=-0.015, p<0.1)$. Não confirmando assim a hipótese proposta. Desta forma, pressupõe-se que em uma sociedade que é caracterizada pela felicidade e alegria, e possui pouca ansiedade com prazos, a capacidade de inovação não é elevada. Hofstede (1980; 2011), elucida que, quando a cultura nacional da indulgência não é proeminente em uma sociedade, e esta é caracterizada pela restrição, este país adota normas sociais rígidas, tem-se mais restrição, e diminui-se a importância do lazer para aquela sociedade.

\section{CONSIDERAÇÕES FINAIS}

Neste estudo buscou-se verificar a influência da cultura nacional sobre a inovação das nações. Para tal, utilizaram-se os scores de inovação dos países como variável dependente e as dimensões: distância de poder, individualismo, masculinidade, incerteza, orientação de longo prazo e indulgência referentes a cultura nacional de Hofstede, como variáveis 
independentes. Para atender ao objetivo, aplicou-se uma pesquisa descritiva, documental e quantitativa. As relações de influência foram analisadas mediante os modelos de regressão linear múltipla.

Os resultados apontaram que, quanto maior a distância de poder menor será a capacidade de inovação da nação, confirmando a $H_{1}$. Para este dado, percebeu-se que a distância do poder influencia negativamente a inovação dos países pela ausência da interação social em diferentes níveis hierárquicos (HOFSTEDE, 1980). Tal fato também pode estar relacionado a países que apresentam altos níveis de centralização e formalização, no que lhe concernem, associam-se as taxas mais baixas de inovação (ZMUD, 1982). Constatou-se também que, quanto maior o nível de individualismo, maior é a capacidade de inovação das nações, confirmando a $H_{2}$. Analisando sob o aspecto da cultura nacional, em países em que a cultura é caracterizada pelo individualismo, as atividades de pesquisa e desenvolvimento tendem a ser maiores do que em países coletivistas (COUTO; VIEIRA, 2004).

Quanto a Hipótese 3, que sugeria que quanto menor o índice de masculinidade maior seria a capacidade de inovação da nação, a hipótese não foi confirmada. Segundo este dado, em nações em que homens e mulheres possuem o poder de forma mais igualitária o índice de inovação foi menor. Deduz-se que nestes países possa haver a separação das atividades por sexo (algumas são realizadas pelo sexo feminino, e outras pelo sexo masculino), e a alternância destes papeis, segundo Hofstede (1980), pode ter contribuído para este resultado.

Com relação à Hipótese 4, verificou-se que quanto maior a incerteza menor é a capacidade de inovação da nação. Supõe-se que os países investigados apresentam leis rígidas, além da presença de um forte formalismo nos negócios, característico de uma sociedade bastante regrada, o que reflete em restrições a atividades inovativas (HOFSTEDE, 1980). E, por fim, a hipótese 5 (quanto maior é o índice de orientação de longo prazo maior é a capacidade de inovação das nações) e a hipótese 6 (quanto maior é a indulgência maior a capacidade de inovação da nação) não foram confirmadas.

No que tange a limitação deste estudo está relacionado ao período de análise, uma vez que, foram utilizados somente os anos mais recentes disponíveis nas bases de dados coletadas. Nesse sentido, um período maior seria interessante. No entanto, mesmo considerando o período utilizado nesta pesquisa, encontrou-se resultados significativos. Apesar desta limitação, entende-se que esta pesquisa pode fomentar futuros estudos, como o uso dos dados de inovação global e cultura nacional no PIB per capita das nações, além da relação destes índices com a corrupção das nações, no sentido de verificar o resultado destas relações. A replicação e ampliação desta pesquisa contribui para o desenvolvimento da área de estudo, tendo em vista a escassez de trabalhos empíricos sobre a influência da cultura nacional no desempenho dos países, tratando-se de inovação. Desta forma, os estudos realizados com esta temática contribuem para o desenvolvimento social e econômico dos países, por meio da geração de informação para a tomada de decisão.

\section{REFERÊNCIAS}

AGUIRRE-BASTOS, C.; WEBER, M. K. Foresight for shaping national innovation systems in developing economies. Technological Forecasting and Social Change, v. 128, p. 186-196, 2017. DOI: https://doi.org/10.1016/j.techfore.2017.11.025.

BANCO MUNDIAL. GDP per capita 2018. Disponível em:

https://data.worldbank.org/indicator/NY.GDP.PCAP.CD. Acesso em: 15 ago. 2018. 
A inovação das nações:

uma análise sob o viés da cultura nacional

BERGER, P. L.; LUCKMANN, T. The social construction of reality. New York: Doubleday, 1967.

BUSZKO, A. T. Cultural implications for the shadow economy. Engineering Economics, v. 29, n. 1, p. 46-52, 2018. DOI: http://dx.doi.org/10.5755/j01.ee.29.1.18069.

COUTO, J. P.; VIEIRA, J. C. National culture and research and development activities. Multinational Business Review, v. 12, n. 1, p. 19-36, 2004. DOI: https://doi.org/10.1108/1525383X200400002

DANIELS, K.; JOHNSON, G.; DE CHERNATONY, L. Task and institutional influences on managers' mental models of competition. Organization Studies, v. 23, n. 1, p. 31-62, 2002. DOI: https://doi.org/10.1177/0170840602231002.

DA SILVA, P. M. F.; MOREIRA, A. National Culture and Its Relationship With Innovation and Corruption. In: Exploring the Influence of Personal Values and Cultures in the Workplace. IGI Global, p. 201-225, 2017. DOI: https://doi.org/10.4018/978-1-5225-2480-9.ch011.

DIMMAGGIO, P.; POWELL, W. The iron cage revisited: Institutional isomorphism and collective rationality in organizational fields. American Sociological Review, v. 48, n. 2, p. 147-160, 1983. DOI: https://doi.org/10.2307/2095101

DOSI, G.; Freeman, C.; NELSON, R. R.; SILVERBERG, G.; SOETE, L. 1988. Technical Change and Economic Theory. Pinter, London.

EDQUIST, C. Systems of Innovation Technologies, Institutions and Organizations. W.W. Norton, London, 1997.

EISENHARDT, K. M. Agency-and institutional-theory explanations: The case of retail sales compensation. Academy of Management journal, v. 31, n. 3, p. 488-511, 1988. DOI: https://doi.org/10.2307/256457.

FÁVERO, L. P.; BELFIORE, P.; SILVA, F. L. da; CHAN, B. L. Análise de dados: modelagem multivariada para a tomada de decisões. Rio de Janeiro: Campus, 2009.

FREEMAN, C. Technology Policy and Economic Performance: Lessons from Japan. Pinter, London, 1987.

FREEMAN, C. Continental, national and sub-national innovation systems-complementarity and economic growth. Research policy, v. 31, n. 2, p. 191-211, 2002. DOI: https://doi.org/10.1016/S0048-7333(01)00136-6.

DUTTA, S.; LANVIN, B.; WUNSCH-VINCENT, S. (Ed.). Global Innovation Index 2017. Cornell University, 2017.

GODIN, B. National innovation system: The system approach in historical perspective. Science, Technology, \& Human Values, v. 34, n. 4, p. 476-501, 2009. DOI: https://doi.org/10.1177/0162243908329187. 
Jandir Tambosi Junior, Silvana Silva Vieira Tambosi e Christian Daniel Falaster

GUAN, J.; CHEN, K. Modeling the relative efficiency of national innovation systems. Research policy, v. 41, n. 1, p. 102-115, 2012. DOI: https://doi.org/10.1016/j.respol.2011.07.001.

HAIR JR, J. F.; BABIN, B.; MONEY, A. H.; PHILLIP, S. Fundamentos de métodos de pesquisa em administração. Porto Alegre: Bookman, 2005.

HOFSTEDE, G. Culture's consequences: international differences in work-related values. Beverly Hills, CA: Sage, 1980.

HOFSTEDE, G. Culture and Organizations. International Studies of Management \& Organization, v. 10, n. 4, p. 15-41, 1980. DOI: https://doi.org/10.1080/00208825.1980.11656300.

HOFSTEDE, G.; BOND, M. H. The Confucius connection: From cultural roots to economic growth. Organizational dynamics, v. 16, n. 4, p. 5-21, 1988. DOI: https://doi.org/10.1016/ 0090-2616(88)90009.

HOFSTEDE, G. Culture's consequences: Comparing values, behaviors, institutions and organizations across nations. Sage publications, 2001.

HOFSTEDE, G.; MINKOV, M. Long-versus short-term orientation: new perspectives. Asia Pacific Business Review, v. 16, n. 4, p. 493-504, 2010. DOI: https://doi.org/10.12691/jbms-26-1. DOI: https://doi.org/10.1080/13602381003637609.

HOFSTEDE, G. Dimensionalizing cultures: The Hofstede model in context. Online readings in psychology and culture, v. 2, n. 1, p. 8, 2011.

HOFSTEDE INSIGHTS. Culture Compass. Disponível em:

https://www.globalinnovationindex.org/analysis-indicator. Acesso em 15 jun. 2018.

HOFSTEDE INSIGHTS. Who we are. 2017. Disponível em: <https://www.hofstedeinsights.com/about-us/>. Acesso em: 09 jun. 2018.

JOHNSON, B.; EDQUIST, C.; LUNDVALL, B. Economic development and the national system of innovation approach. In: FIRST GLOBELICS CONFERENCE. 1, 2003, Rio de Janeiro. Anais... Rio de Janeiro: Redesist, 2004, p. 1-24.

KOSTIS, P. C.; KAFKA, K. I.; PETRAKIS, P. E. Cultural change and innovation performance. Journal of Business Research, v. 88, p. 306-313, 2018. DOI:

https://doi.org/10.1016/j.jbusres.2017.12.010.

KRISTJÁNSDÓTTIR, H.; GUĐLAUGSSON, P. Ö.; GUĐMUNDSDÓTTIR, S.; AĐALSTEINSSON, G. D. Hofstede national culture and international trade. Applied Economics, v. 49, n. 57, p. 57925801, 2017. DOI: https://doi.org/10.1080/00036846.2017.1343446.

KWOK, C. CY; TADESSE, S. National Culture and Financial Systems. Journal of International 
A inovação das nações:

uma análise sob o viés da cultura nacional

Business Studies, v. 37, n. 2, p. 227-247, 2006. DOI:

https://doi.org/10.1057/palgrave.jibs.8400188.

LUNDVALL, B. National Systems of Innovation: Towards a Theory of Innovation and Interactive Learning. Pinter, London, 1992.

LUNDVALL, B. The Learning Economy and the Economics of Hope. Anthem Press, 2017.

MAROCO, J. Análise estatística: com utilização do SPSS. Lisboa: Abril, 2003.

MEYER, J. W.; ROWAN, B. Institutionalized organizations: Formal structure as myth and ceremony. American Journal of Sociology, v. 83, n. 2, p. 340-363, 1977. DOI:

http://dx.doi.org/10.1590/0034-7612155117.

MEYER, J. W.; SCOTT, W. R.; TERRENCE, E. Deal. Institutional and techmical sources of organizational structure of educational organizations. In: MEYER, J. W.; SCOTT, W. R. (Ed.) Organizational environments: ritual and rationality. Beverly Hills, CA: Sage, p. 45-70, 1983. DOI: https://doi.org/10.1002/pam.4050030417.

NELSON, R. R. National innovation systems: a retrospective on a study. Industrial and Corporate Change, v. 1, n. 2, p. 347-374, 1992. DOI: https://doi.org/10.1093/icc/1.2.347.

NELSON, R. R. National Innovation Systems: A Comparative Analysis. Oxford University Press, 1993.

NELSON, R. R.; NELSON, K. Technology, institutions, and innovation systems. Research Policy, v. 31, n. 2, p. 265-272, 2002. DOI: https://doi.org/10.1016/S0048-7333(01)00140-8.

NORTH, D. C. Institutions. Journal of Economic Perspectives, v. 5, n. 1, p. 97-112, 1991. DOI: https://doi.org/10.1257/jep.5.1.97.

OSTAPENKO, N. National culture, institutions and economic growth: The way of influence on productivity of entrepreneurship. Journal of Entrepreneurship and Public Policy, v. 4, n. 3, p. 331-351, 2015. DOI: https://doi.org/10.1108/JEPP-11-2014-0040.

PRADHAN, R. P.; ARVIN, M. B.; BAHMANI, S. Are innovation and financial development causative factors in economic growth? Evidence from a panel granger causality test. Technological Forecasting and Social Change, v. 132, p. 130-142, 2018. DOI: https://doi.org/10.1016/j.techfore.2018.01.024.

ROSSETTO, C. R.; ROSSETTO, A. M. Teoria institucional e dependência de recursos na adaptação organizacional: uma visão complementar. RAE-eletrônica, v. 4, n. 1, 2005. DOI: https://doi.org/10.1590/S1676-56482005000100010.

SALZMANN, A.; SOYPAK, K. National culture and private benefits of control. . Finance Research Letters, v. 20, p. 199-206, 2017. DOI: https://doi.org/10.1016/j.frl.2016.09.027. 
SELZNICK, P. Institucionalism "Old" and "New". Administrative Science Quarterly. v. 41, n. 2, p. 270-277, jun. 1996. DOI: https://doi.org/10.2307/2393719.

SHARIPOV, I. Exogenous vs Endogenous Growth in the EU's EaP and Central Asian Countries. Scientific Annals of Economics and Business, v. 63, n. s1, p. 109-124, 2016. DOI: https://doi.org/10.1515/saeb-2016-0138.

THE GLOBAL INNOVATION INDEX. View 2017 analysis. Disponível em: https://www.globalinnovationindex.org/analysis-indicator. Acesso em 15 maio. 2018.

THE WORLD BANK. GDP per capita. Disponível em: https://data.worldbank.org/indicator/NY.GDP.PCAP.CD. Acesso em: 15 maio. 2018.

ZHAO, X.; LI, H.; RAUCH, A. Cross-country differences in entrepreneurial activity: The role of cultural practice and national wealth. Frontiers of Business Research in China, v. 6, n. 4, p. 447-474, 2012. DOI https://doi.org/10.3868/s070-001-012-0021-0.

ZMUD, R. W. Diffusion of modern software practices: influence of centralization and formalization. Management science, v. 28, n. 12, p. 1421-1431, 1982. DOI: http://dx.doi.org/10.1287/mnsc.28.12.1421. 